\title{
COMPARAÇÃO DO DESENVOLVIMENTO NEUROMOTOR DE LACTENTES PREMATUROS COM E SEM HEMORRAGIA PERI-INTRAVENTRICULAR
}

\author{
COMPARING THE NEUROMOTOR DEVELOPMENT OF \\ PREMATURE INFANTS WITH AND WITHOUT PERIIIN- \\ TRAVENTRICULAR HEMORRHAGE
}

Bruna Kelly Ferreira (ORCID: 0000-0001-8808-471X) ${ }^{1}$ Dayane Duarte Abreu (ORCID: 0000-0001-5736-070X) ${ }^{1}$ Jadiane Dionísio (ORCID: 0000-0002-8734-4089)

Autor Correspondente

Jadiane Dionísio

E-mail: jadydionisio@gmail.com.

${ }^{1}$ Curso de Fisioterapia. Universidade Federal de Uberlândia.

\begin{abstract}
RESUMO
Introdução: lactentes pré-termo podem apresentar imaturidade funcional por não completar o desenvolvimento intrauterino, assim como complicações neurológicas relacionadas a Hemorragia Peri-intraventricular (HPIV). Sendo assim, tanto a prematuridade quanto a presença de HPIV influenciam diretamente no desenvolvimento motor do lactente, gerando atrasos nas aquisições ou até mesmo habilidades atípicas. Objetivo: avaliar e comparar o desenvolvimento neuromotor grosso dos lactentes prematuros com e sem diagnóstico de HPIV. Métodos: foi realizado um estudo prospectivo, transversal e avaliativo. A amostra foi por conveniência composta por 20 lactentes de 0 a 18 meses, prematuros com e sem diagnóstico de HPIV conforme os critérios de inclusão. Os lactentes foram avaliados por uma ficha estruturada e pela escala de desenvolvimento Alberta Infant Motor Scale (AIMS), nas posturas prono, supino, sentado e em pé. Resultados: foi observado que lactentes com diagnóstico de HPIV obtiveram menor pontuação na escala AIMS, assim como a relação entre menor peso e a idade gestacional com a menor pontuação da escala, independentemente do grupo. Ademais, verificou correlação negativa entre menor peso e menor a idade gestacional quanto à presença de hemorragia cerebral. Conclusões: lactentes prematuros com diagnóstico de hemorragia cerebral apresentam maior atraso no desenvolvimento neuromotor grosso quando comparado com aqueles sem diagnóstico de HPIV. Da mesma forma, a idade e o peso estão diretamente relacionados com o atraso do desenvolvimento motor normal e presença de HPIV.
\end{abstract}

Palavras-chave: Hemorragia Cerebral; Lactente; Prematuro; Desenvolvimento Infantil; Desempenho Psicomotor.

\begin{abstract}
Objective: preterm infants may have functional immaturity due to not completing intrauterine development, as well as neurological complications related to Peri-Intraventricular Hemorrhage (PIVH). Therefore, both prematurity and the presence of PIVH directly influence the infant's motor development, generating delays in acquisitions or even atypical skills. Objective: to assess and compare gross neuromotor development of premature infants with and without a PIVH diagnosis. Methods: a prospective, evaluative cross-sectional study was performed. The convenience sample was composed of 20 premature infants aged between 0 to 18 months with and without a PIVH diagnosis, in accordance to the inclusion criteria. The assessment of the infants was conducted using a structured form and the Alberta Infant Motor Scale (AIMS), in prone, supine, sitting, and standing positions. Results: infants with a PIVH diagnosis scored lower on the AIMS. Moreover, results showed that lower body weight and lower gestational age were associated with lower scores on the scales, regardless of the group. Lower body weight and lower gestational age also negatively correlated with the presence of brain hemorrhage. Conclusion: premature infants with a brain hemorrhage diagnosis showed a delayed gross neuromotor development, compared to those without a PIVH diagnosis. Thus, age and body weight are directly associated to delays on the normal motor development and the presence of PIVH.
\end{abstract}

Keywords: Brain Hemorrhage; Infant; Premature; Child Development; Psychomotor Performance. 


\section{INTRODUÇÃO}

Os lactentes prematuros, aqueles abaixo de 37 semanas de gestação ${ }^{1}$, geralmente, não atingem a maturidade funcional e estrutural dos órgãos e tecidos, visto que não completaram o desenvolvimento intrauterino, levando a possíveis alterações e atrasos no desenvolvimento neuromotor quando comparados aos lactentes nascidos a termo ${ }^{2}$. Os partos prematuros podem ocorrer devido a multifatores ou serem idiopáticos. Os principais fatores de risco estão relacionados com baixa assistência pré-natal ou quando esta é inadequada; pré-eclâmpsia; baixo nível socioeconômico; anemia e doenças maternas; gestação múltipla; infecções; complicações durante o parto; hemorragias e sofrimento fetal ${ }^{3}$.

O lactente pré-termo, por apresentar imaturidade dos sistemas, é mais suscetível ao aparecimento de complicações e deficiências físicas, neurológicas e cognitivas, podendo acarretar atraso ou atípico processo em sua evolução ${ }^{3}$. Dentre essas complicações, destacam-se: hemorragia intracraniana; anóxia; doença de membranas hialinas; Comunicação Intra-Atrial (CIA); Persistência do Canal Arterial (PCA); e sepse ${ }^{1}$.

A Hemorragia Peri-intraventricular (HPIV) é uma das complicações neurológicas que acometem o Recém-Nascido Pré-Termo (RNPT), principalmente naqueles de muito baixo peso ${ }^{4}$. A HPIV acontece devido a imaturidade da matriz germinativa ${ }^{5}$, e provável mudança do fluxo sanguíneo cerebral com rompimento dos vasos, por causa da dificuldade de autorregulação cardiovascular do RNPT ${ }^{4-5}$. Além dos fatores peso e idade gestacional, há outros que estão relacionados com a HPIV, como a baixa pontuação no índice de Apgar no primeiro e no quinto minuto, apneia, pneumotórax, acidose, hipercapnia, presença de sepse neonatal, necessidade de ventilação mecânica, entre outros ${ }^{6,7}$.

A HPIV possui quatro graduações, sendo os graus III e IV propensos a gerar graves dificuldades na aprendizagem, e grandes alterações no desenvolvimento neuropsicomotor e somatossensorial (tais como hipóxia e paralisia cerebral), até mesmo a mortalidade ${ }^{6,8-10}$.

Visto isso, acredita-se que a prematuridade e a presença de HPIV influenciam diretamente no desenvolvimento neuropsicomotor do lactente. Esse desenvolvimento caracteriza-se pela mudança nas habilidades neurais, psíquicas e motoras ao longo da vida, que resulta da interação entre processos biológicos geneticamente determinados e ambientais ${ }^{2}$. O lactente adquire movimentos e posturas conforme o estímulo oferecido e as necessidades existentes, tornando-o cada vez mais eficientes em determinadas funções.

De acordo com as complicações e atrasos advindos da prematuridade e da presença da HPIV, é imprescindível a necessidade de uma adequada avaliação fisioterapêutica precoce, corroborado por Santos ${ }^{11}$ que comprovou a importância da Escala Motora Infantil de Alberta (Alberta Infant Motor Scale - AIMS) na identificação dos atrasos motores, permitindo assim o encaminhamento precoce para fisioterapia e facilitando o desenvolvimento futuro dos lactentes.

A AIMS é uma escala que avalia o desenvolvimento neuromotor de lactentes (0 a 18 meses) incorporando o conceito neuromaturacional e a Teoria dos Sistemas Dinâmicos. Isso ocorre, pois a escala avalia as habilidades motoras por meio de observação de movimentos antigravitacionais de forma voluntária ${ }^{12}$. Essa escala foi desenvolvida e validada por Piper e $\operatorname{Darrah}^{13}$; e mesmo não sendo referência para avaliação de lactentes com HPIV, esta é padrão ouro para avaliação e identificação de atraso do desenvolvimento motor, sendo muito utilizada na prática clínica. Desse modo, a escala consegue identificar precocemente os atrasos e direcionar para um tratamento mais adequado.

Supõe-se que a baixa idade gestacional e o baixo peso possam influenciar no desencadeamento da hemorragia cerebral. Ademais, acredita-se que esses dois fatores intrínsecos comprometam o desenvolvimento neuromotor dos lactentes independentemente da presença de hemorragia cerebral. Entretanto, acredita-se que os lactentes prematuros com diagnóstico de HPIV tenham maior atraso neuromotor em relação àqueles apenas prematuros, devido à soma dos fatores complicadores. Para tanto, o objetivo deste estudo foi avaliar e comparar o desenvolvimento neuromotor grosso dos lactentes prematuros com e sem diagnóstico de HPIV. 


\section{MÉTODOS}

Foi realizado um estudo prospectivo, transversal, observacional e avaliativo. A população do estudo foi selecionada por conveniência, sendo de lactentes prematuros nascidos no ano de 2018 e 2019 , que realizam fisioterapia, e aqueles que estavam na lista de espera para o tratamento na clínica de Fisioterapia Universitária.

A partir de então, foram selecionados: lactentes com idade cronológica entre 0 e 18 meses, prematuros (idade gestacional até 36 semanas e 6 dias) com e sem o diagnóstico de Hemorragia Peri-intraventricular, de ambos os sexos e etnias. Não foram incluídos lactentes com alterações genéticas.

Dessa forma, obtiveram-se 20 lactentes prematuros, os quais foram agrupados em dois grupos com 10 lactentes. $\mathrm{O}$ grupo 1 foi composto de lactentes prematuros com diagnóstico de HPIV; e outro grupo, com 10 lactentes prematuros sem diagnóstico de HPIV. Dos 20, 11 eram do sexo feminino, e 9, do sexo masculino.

Inicialmente, foi realizado o preenchimento de uma ficha de avaliação estruturada (quesitos como: data de nascimento, idade cronológica, idade gestacional, via de parto, peso ao nascer; ausência ou presença de HPIV; se realiza tratamento fisioterapêutico; se frequenta creches ou escolas; entre outros) e do questionário socioeconômico, o qual avaliou questões relacionadas com os pais e/ou responsáveis, a saber: nível de escolaridade; situação conjugal; renda familiar mensal, entre outros. Após, foi realizada a avaliação dos lactentes por meio da AIMS, com duração máxima de uma hora.

A escala AIMS, desenvolvida por Piper e Darrah ${ }^{13}$, observa as aquisições motoras do Recém-Nascido (RN) até o andar independente do lactente (aproximadamente 18 meses). São 58 itens em 4 posições diferentes: prono (21 itens), supino (9 itens), sentado (12 itens) e em pé (16 itens). Durante a avaliação, observa-se o comportamento motor do lactente, levando em consideração aspectos como sustentação do peso, postura e movimentos antigravitacionais de acordo com a sua idade. Conforme os movimentos apresentados no repertório do lactente, para cada item observado ou não observado, registravam-se as pontuações 1 e 0 respectivamente. Ao final, os itens observados são somados, resultando em quatro subtotais e transformados em percentis variando de $5 \%$ a $90 \%{ }^{2,1}$.

Ressalta-se ainda que as coletas de dados foram executadas por dois pesquisadores previamente treinados, com índice de concordância interpesquisadores maior que $90 \%$, em uma sala reservada na Clínica de Fisioterapia Universitária, constando com iluminação e temperatura ambiental adequadas, colchões e tatames de EVA, e brinquedos adequados para a idade.

Os resultados coletados em forma física foram então transferidos para uma tabela no computador, em que foi aplicado o teste paramétrico Teste $t$ de Student para comparação dos grupos com e sem diagnóstico de HPIV em relação à pontuação da escala (variável categórica), e Correlação de Pearson para peso e idade gestacional com pontuação da AIMS e presença de HPIV, por meio do pacote estatístico Bioestatic 5.5. Para as variáveis contínuas (peso, altura e entre outros), foram utilizados média e desvio-padrão; e para as variáveis categóricas (idade, dados maternos, posturas da escala e entre outros), utilizou-se a porcentagem.

Este trabalho foi aprovado pelo Comitê de Ética em Pesquisa em Seres Humanos (CAAE: 30218620.0.0000.5152) e seguiu todos os preceitos éticos da Resolução ${ }^{\circ}$ 466/12, assim como o consentimento dos pais ou responsáveis legais mediante assinatura do Termo de Consentimento Livre e Esclarecido.

\section{RESULTADOS}

Dos 20 lactentes, 9 (45\%) eram do sexo masculino; e 11 (55\%), do sexo feminino. O grupo 1 foi composto por quantidades iguais de ambos dos sexos; enquanto no grupo 2, havia 4 do sexo masculino e 6 do sexo feminino. No grupo 1, 5 lactentes $(50 \%)$ apresentavam diagnóstico de hemorragia unilateral; e os outros $5(50 \%)$, bilateral.

Em relação à realização do 


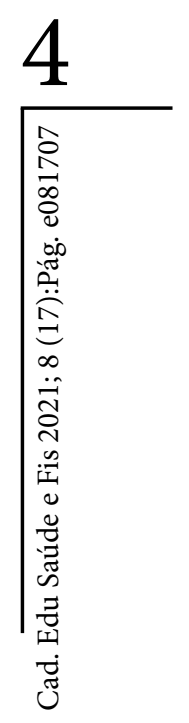

tratamento fisioterapêutico, no grupo 1, havia 6 lactentes que realizavam fisioterapia; enquanto no grupo sem hemorragia, havia apenas 2 lactentes.

Quanto ao peso ao nascer e a idade gestacional, a média e o desvio-padrão do grupo 1 foram, respectivamente, 1.332 gramas $\pm 531,3$ gramas e 29,1 semanas $\pm 2,668$ semanas. No grupo 2 , as médias obtidas foram de 2.015,5 gramas $\pm 753,4$ gramas e 33,3 semanas $\pm 3,755$ semanas.

Em relação à análise individual das posturas pela escala AIMS, obtiveram-se os resultados a seguir. Na postura prono, o grupo 1 teve pontuação média de 5,1 (23,8\%); e o grupo 2, de 16,7 (80,9\%).
Em supino, o grupo 1 teve média de 5,4 $(55,6 \%)$; e o grupo 2 , de $8,4(88,9 \%)$. Na postura sentada, o grupo 1 obteve média de $1,9(16,7 \%)$; e o grupo 2 , de $8,5(75 \%)$. Na postura em pé, o grupo 1 pontuou a média de 2,5 (18,5\%); e grupo 2, de $46(75 \%)$. Por fim, o score total do grupo 1 foi de $17,5 \%$; e do grupo 2 , de $54,7 \%$.

Ao comparar a avaliação feita com a AIMS em lactentes prematuros com diagnóstico de HPIV e aqueles sem o diagnóstico, observaram-se diferenças significativas (p:0,004), sendo que os lactentes prematuros com HPIV obtiveram menor pontuação na escala (figura 1).

Figura 1. Comparação entre pré-termos (PT) com e sem HPIV de acordo com escore da AIMS. Uberlândia, 2020

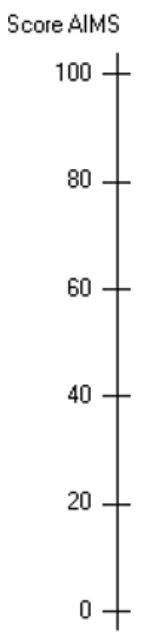

Comparação do Score da Escala AlMS

$00+$
$80+$
$60-$
$40-$
$20-$
$0+$

Entre Pré-termo com e sem HPIV 
Quanto à análise intergrupo, foi realizada a correlação entre peso, idade gestacional e pontuação da AIMS; sendo observado no grupo de lactentes sem HPIV correlação negativa (r:0.710; p: 0,0004), indicando que quanto menor o peso do lactente, menor a pontuação na escala (figura 2), assim como quanto menor a idade gestacional (menor que 30 semanas), menor a pontuação. (r: 0.797; p: <0,0001) (figura 3). A mesma situação foi observada na correlação intergrupo com HPIV, sendo a correlação negativa entre menor peso ( $r: 0.483 ; p: 0,03)$ e presença de hemorragia cerebral (figura 4); assim como menor idade gestacional (r: 0,$553 ; \mathrm{p}: 0,01) \mathrm{com}$ HPIV (figura 5).

Figura 2. Correlação Peso ao nascer com score AIMS. Uberlândia, 2020

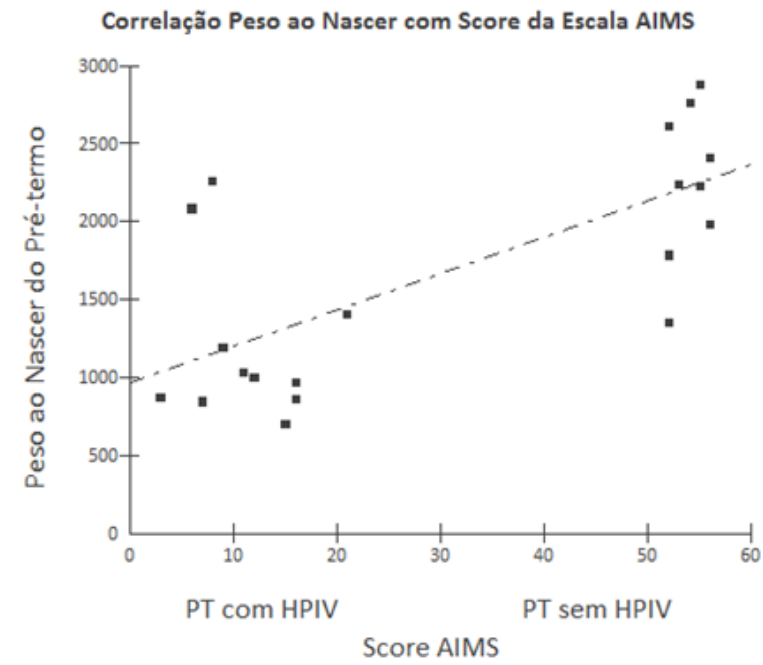

Figura 4. Correlação Peso ao nascer com presença de HPIV. Uberlândia, 2020

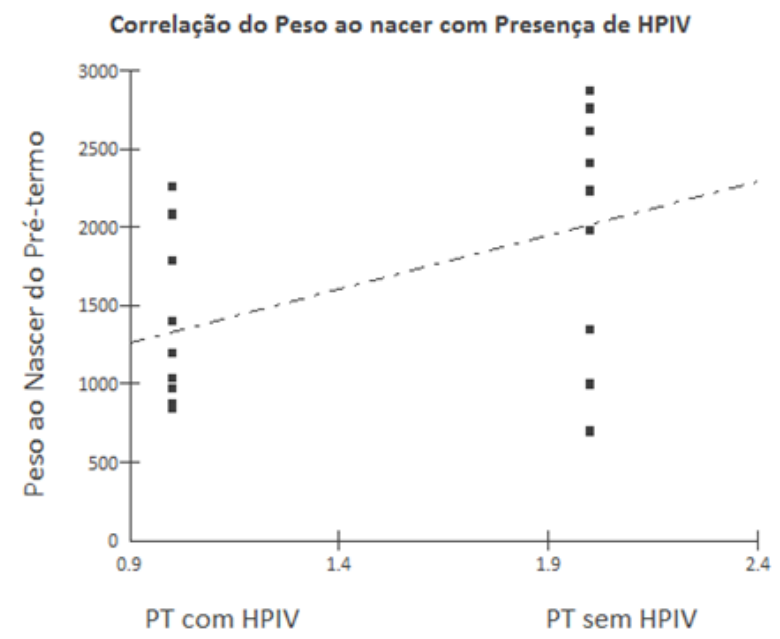

Presença de HPIV
Figura 3. Correlação Idade Gestacional com score AIMS. Uberlândia, 2020

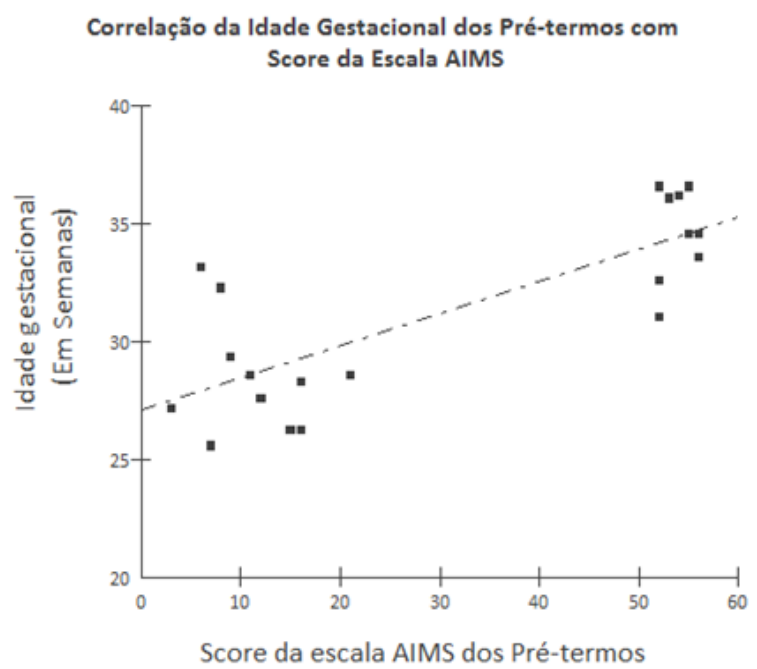

Figura 5. Correlação da Idade Gestacional de pré-termos com e sem HPIV. Uberlândia, 2020

Correlação da Idade Gestacional dos Pré-termos com Presença de HPIV

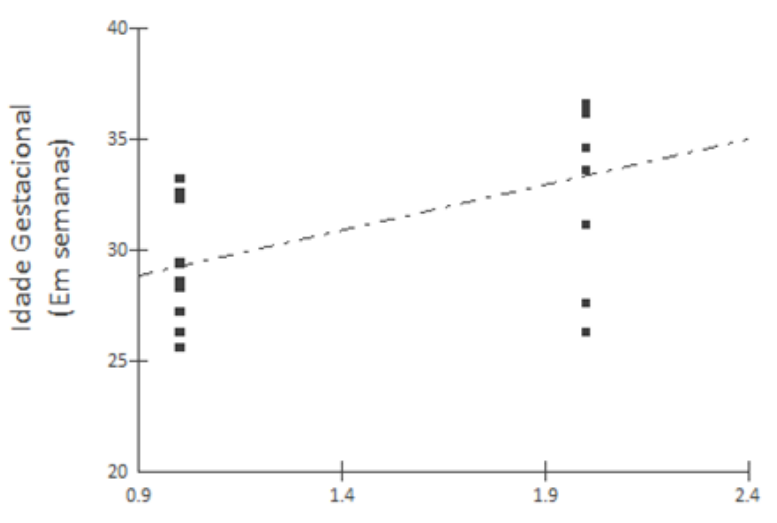

PT com HPIV

PT sem HPIV

Presenca de HPIV 


\section{DISCUSSÃO}

O presente estudo demonstra que o grupo 1 (prematuros com diagnóstico de HPIV) obteve menor pontuação na escala AIMS, tanto no score total quanto na avaliação individual das posturas (prono, supino, sentado e em pé), corroborando, assim, a terceira hipótese levantada. Esse cenário se dá por consequência da ação da HPIV no sistema nervoso central imaturo do prematuro, uma vez que este pode causar destruição de precursores das células gliais que estão em processo de migração para regiões corticais, ocasionando assim prejuízo no desenvolvimento neuropsicomotor ${ }^{15}$.

Corroborando os achados, Airoldi et al. ${ }^{16}$ ao utilizar o teste neurológico Dubowitz e Dubowitz modificado, o qual tem por objetivo avaliar o desenvolvimento neuropsicomotor do lactente prematuro, observaram que os lactentes com Muito Baixo Peso ao Nascer (MBPN) e com diagnóstico de HPIV e/ou Leucomalacia Periventricular (LPV) apresentaram menor desempenho quando comparados com outros sem o diagnóstico. Além disso, os autores aventaram a associação direta de idade gestacional e peso ao nascer com diagnóstico de HPIV e/ou LPV, sendo observados resultados similares neste estudo.

A correlação da baixa idade gestacional com a maior incidência de HPIV pode ser atribuída às ações imaturas do sistema nervoso central ao final do segundo trimestre gestacional. A matriz germinativa possui apenas uma parede endotelial ainda imatura e frágil com intensa atividade metabólica. Desse modo, a vulnerabilidade anatômica associada à imaturidade de autorregulação vascular nesse período podem contribuir para uma maior suscetibilidade da matriz à hemorragia em resposta das flutuações na pressão cardiovascular $^{16}$.

Ahn et al. ${ }^{17}$, em seu estudo, obtiveram maior incidência de HPIV em lactentes nascidos com menos de 22 semanas gestacionais, sendo observado tal resultado também em Baixo Peso ao Nascer (BPN), contudo, a idade gestacional apresentou-se como fator mais significativo entre eles. Corroborando o estudo anterior, Marret et al. ${ }^{18}$ trazem o nascimento prematuro, principalmente naqueles lactentes com menor idade gestacional, como um risco elevado para a ocorrência da HPIV e déficit neurológico, reforçado por Rosário et al. ${ }^{19}$, os quais observaram que, assim como a idade gestacional, o peso ao nascer está relacionado com o desenvolvimento de HPIV - sendo que idade gestacional $\leq 32$ semanas apresentou 16,5 vezes mais risco de ter HPIV; e o BPN ( $\leq 1500 \mathrm{~g})$, um risco 6,68 vezes maior de serem acometidos pela HPIV.

Tais achados supracitados vem ao encontro a este, o qual indica associação entre BPN do lactente a maior chance de HPIV. Ribeiro et al..$^{20}$ explicam que o nascimento prematuro pode interferir, principalmente, nas fases de multiplicação glial, migração neuronal e sua organização, o que pode possibilitar a alteração na organização cerebral, sendo a imaturidade cerebral precoce associada ao nascimento prematuro e o peso do lactente determinantes para o desenvolvimento infantil.

Estudos sobre incidência de HPIV em lactentes RN e a relação com o peso ao nascer mostram que a incidência de HPIV varia inversamente naqueles com $\mathrm{MBPN}^{17,21}$. Além disso, em seu estudo, Moreira et al. ${ }^{22}$ observaram que quase um quarto dos $\mathrm{RN}$ apresentaram alguma morbidade, com maior prevalência nos nascidos com MBPN, e afirmaram que quanto menor o peso ao nascer, maior o risco de comorbidades, entre elas, a HPIV. Para tanto, inferimos a primeira hipótese deste estudo, tendo em vista que quanto menor a idade gestacional e o peso ao nascer, maior é o risco de ocorrência da HPIV.

Assim como a HPIV, pudemos observar associação das variáveis: BPN e idade gestacional inferior a 30 semanas, com a menor pontuação na AIMS em ambos os grupos.

Guimarães e colaboradores ${ }^{23}$, em objetivo similar a este estudo, porém utilizando a escala de avaliação Test of Infant Motor Performance (TIMP), verificaram que prematuros com média de idade gestacional de 31,1 semanas e peso médio de 1.424 gramas apresentaram desempenho motor significativamente inferior quando comparados ao grupo RN a termo. 
Acredita-se que essas diferenças podem ser observadas uma vez que os prematuros se encontram com o tempo reduzido no ambiente intrauterino e maior exposição a fatores estressantes da Unidade de Terapia Intensiva Neonatal (Utin). Outros fatores que podem influenciar nos atrasos seriam a redução do tônus muscular e a ação da gravidade sobre a musculatura fraca dos prematuros.

Embora a tendência do tônus muscular flexor seja aumentar à medida que o prematuro se desenvolve, ele não consegue atingir o grau completo do tônus muscular do lactente a termo. Assim, influencia no equilíbrio entre grupos musculares flexores e extensores que poderão interferir em futuras aquisições motoras ${ }^{24}$.

Para Formiga ${ }^{25}$, ao utilizar também a escala TIMP, verificou-se que os lactentes com MBPN (<1500 g) novamente apresentaram maior atraso em relação àqueles de baixo peso, comprovando, assim, que a imaturidade funcional e estrutural dos órgãos e tecidos pode levar a um desenvolvimento motor diferente dos lactentes nascidos a termo, associado a fatores de risco biológico.

Outros autores também evidenciam que a prematuridade, especialmente quando associada ao baixo peso ao nascimento, pode interferir na estabilidade e no ritmo de aquisição de padrões motores durante o primeiro ano de vida dos lactentes, sugerindo que quanto menor o peso e idade gestacional no nascimento, maior a chance de atraso. Ademais, a prematuridade pode levar à dificuldade para entender ou processar as informações sensoriais para o controle do movimento ${ }^{26,27}$.

Apesar dos resultados relevantes, identificamos como fator limitador a amostra pequena, fato este que ocorreu devido a dificuldades de agenda e interesse dos responsáveis, assim como a ausência de informação sobre frequência e assiduidade em relação ao tratamento fisioterapêutico, apesar desta não ser variável de análise neste estudo.

\section{CONCLUSÕES}

Concluímos que os lactentes prematuros com diagnóstico de hemorragia cerebral apresentam maior atraso no desenvolvimento neuromotor grosso quando comparados com aqueles que não possuem diagnóstico de HPIV. Além disso, os lactentes que nasceram antes de 30 semanas gestacionais e com baixo peso manifestaram menor pontuação na escala e correlação positiva com HPIV.

Desse modo, o estudo permite identificar os atrasos neuromotores de lactentes prematuros com e sem HPIV e redirecionar medidas terapêuticas mais eficazes, possibilitando a antecipação das ações de intervenção, reduzindo os riscos de sequelas futuras.

\section{REFERÊNCIAS}

1. Zomignani AP, Zambelli HJL, Antonio MA. Desenvolvimento cerebral em recém-nascidos prematuros. Rev Paulista Pediatria. 2009;27(2):198-203.

2. Maia PC, Silva LP, Oliveira MMC, Cardoso MV. Desenvolvimento motor de crianças prematuras e a termo - uso da Alberta Infant Motor Scale. Acta Paul Enferm. 2011;24(5):670-675.

3. Medeiros JKB, Zanin RO, Alves KS. Perfil do desenvolvimento motor do prematuro atendido pela Fisioterapia. Rev Soc Bras Clín Méd. 2009;7:367-372.

4. Abreu LC, Souza AMB, Oliveira AG, Selestrin CC, Guerra MSB, Ferreira C, et al. Incidência de hemorragia peri-intraventricular em recém-nascidos pré-termo e a relação com o peso ao nascer. Rev Bras Crescimento Desenvolv Hum. 2007;17(2):24-30.

5. Jorch G, Jorch N. Failure of auto regulation of cerebral blood flow in neonates studies by pulsed Doppler ultrasound of internal carotid artery. Eur J Pediatr. 1987;146(5):468-72.

6. Redondo F, Falcó O, Rodriguez A, Vicente C, Carande M. Hemorragia 
intracraneana del prematuro, frecuencia de presentación y factores de riesgo. Arch Arg Pediatr. 2003;101(4):256-60.

7. Khodapanahandeh F, Khosravi N, Larijani T. Risk factors for intraventricular hemorrhage in very low birth weight infants in Tehran, Iran. Turk J Pediatr. 2008; 50(3): 247-52.

8. Segre CA, Costa HP, Lippi UG. Perinatologia: fundamentos e prática. 3. ed. São Paulo: Sarvier; 2009.

9. Mendonza AM, Kalil LF, Moog JC, Hernández AG, Ochoa JW, Hidalgo YS. Evaluación de la incidência y los factores de riesgo para hemorragia intraventricular (HIV) en la cohorte de recién nacidos prematuros atendidos en la unidade neonatal del Hospital Universitario San Vicente de Paúl, de Meddellín, en el período comprendido entre enero de 1999 y diciembre de 2004. IATREIA. 2007;20(4):341-53.

10. Roze E, Kerstjens JM, Maathuis CG, Horst HJ, Bos AF. Risk factors for adverse outcome in preterm infants with periventricular hemorrhagic infarction. Pediatrics. 2008;122(1):46-52.

11. Santos RS, Araújo AP, Porto MA. Early diagnosis of abnormal development of preterm newborns: assessment instruments. J Pediatr. 2008;84(4):289-299.

12. Manacero S. Desempenho motor de prematuros durante o primeiro ano de vida na Escala Motora Infantil de Alberta (AIMS) [dissertação]. Porto Alegre (RS): Pontífícia Universidade Católica do Rio Grande do Sul; 2005

13. Piper MC, Pinnell LE, Darrah J, Maguile T, Byrne PJ. Construction and validation of the Alberta Infant Motor Scale (AIMS). Can J Public Health. 1992;83(suppl 2):46-50.

14. Manacero S. Desempenho motor de prematuros durante o primeiro ano de vida na Escala Motora Infantil de Alberta (AIMS) [dissertação]. Porto Alegre (RS): Pontifícia Universidade Católica do Rio Grande do Sul; 2005.

15. Tavolone MGG. Hemorragia
Peri-intraventricular em recém-nascidos pré-termo com restrição de crescimento intrauterino [dissertação]. Uberlândia (MG): Universidade Federal de Uberlândia; 2014.

16. Airoldi MJ, Silva SBC, Souza RCT. Avaliação de recém nascidos pré-termo com hemorragia peri-intraventricular e/ou leucomalácia periventricular. Rev Neurocienc. 2009; 17(1):24-29

17. Ahn SY, Shim S-Y, Sung IK. Intraventricular Hemorrhage and Post Hemorrhagic Hydrocephalus among Very-Low-Birth-Weight Infants in Korea. J Korean Med Sci. 2015;30(Suppl 1):S52.

18. Marret S, Marchand-Martin L, Picaud J-C, Hascoët J-M, Arnaud C, Rozé $\mathrm{J}-\mathrm{C}$, et al. Brain Injury in Very Preterm Children and Neurosensory and Cognitive Disabilities during Childhood: The EPIPAGE Cohort Study. PLoS One. 2013;8(5):e62683.

19. Rosário MJP, Lorenzo CM, Sonza A, Parcias SR. Fatores de risco perinatais e avaliação neuropsicomotora em crianças pré-termo com e sem hemorragia peri-intraventricular. ConScientiae Saúde. 2019;18(1):116-124.

20. Ribeiro CC, Pachelli MRO, Amaral NCO, Lamônica DAC. Habilidades do desenvolvimento de crianças prematuras de baixo peso e muito baixo peso. CoDAS. 2017;29(1):e20160058.

21. Radic JAE, Vincer M, McNeely PD. Outcomes of intraventricular hemorrhage and posthemorrhagic hydrocephalus in a population-based cohort of very preterm infants born to residents of Nova Scotia from 1993 to 2010. J Neurosurg Pediatr. 2015;15(6):580-8.

22. Moreira RS, Magalhães LC, Alves CRL. Effect of preterm birth on motor development, behavior, and school performance of school-age children: a systematic review. J Pediatr (Rio J). 2014;90(2):119-34.

23. Guimarães CLN, Reinaux CM, Botelho ACG, Lima GMS, Filho JEC. Desenvolvimento motor avaliado pelo Test of Infant 
Motor Performance: comparação entre lactentes pré-termo e a termo. Rev Bras Fisioter. 2011;15(5):357-62.

24. Sampaio TF, Nogueira KPA, Pontes TB, Toledo AM. Comportamento motor de lactentes prematuros de baixo peso e muito baixo peso ao nascer. Fisioter Pesq. 2015;22(3):253-260.

25. Formiga CKMR. Detecção de riscos para problemas no desenvolvimento de bebês nascidos pré-termo no primeiro ano de vida [tese]. São Paulo (SP): Faculdade de Medicina de Ribeirão Preto da Universidade de São Paulo; 2009.

26. Van Haastert IC, de Vries LS, Helders PJ, Jongmans MJ. Early gross motor development of preterm infants according to the Alberta Infant Motor Scale. J Pediatr. 2006;149(5):617-22.

27. Erikson C, Allert C, Carlberg EB, Katz-Salamon M. Stability of longitudinal motor development in very low birth weight infants from 5 months to 5.5 years. Acta Paediatr. 2003;92(2):197-203. 